\title{
VISUAL FIXATION PATTERN IN OPTOKINETIC NYSTAGMUS
}

\author{
Masaaki KITAHARA and Takashi FuTAKI
}

\section{INTRODUCTION}

In order to see a moving object clearly, its image must be accurately maintained on the fovea of the eye at least intermittently. For optokinetic nystagmus, this is thought to be achieved by visual fixation during the slow phase of nystagmus (ter Braak ${ }^{1} 1936$ ).

Until recently, however, it was difficult to distinguish whether or not the image of the moving object was fixed on the fovea. Thus in most reports, optokinetic nystagmus has been evaluated indirectly according to its frequency, amplitude, or regularity, etc. (Ehlers $^{3}$ 1926, Fox ${ }^{5}$ 1926, Gruttner ${ }^{7}$ 1936, Mckensen ${ }^{9,11}$ 1954, 1960, Ohm ${ }^{14}$ 1957, Enocksson ${ }^{4}$ 1956, etc.).

The authors have here attempted to clarify the patterns of visual fixation i.e. to show the dynamic correlation between the gaze point and the moving object in optokinetic nystagmus. To accomplish this, a gaze point indicator was superimposed upon the image of viewed objects; and optokinetic nystagmus was observed as moved at various frequencies and velocities. A discussion of the adaptability and complexities of optokinetic nystagmus is also included.

\section{EXPERIMENTAL METHODS}

1. The method for superimposing the gaze point indicator in the field of vision:

The gaze point in the visual scene was determined by using an Eye Mark Recorder (NAC) mounted on the head of the test subject as shown in Figure 1.

A schematic diagram of the Eye Mark Recorder is shown in Figure 2. A spot lamp fixed laterally at the front of the eye projects a beam of light onto the cornea. The light reflected from the cornea is reflected upward by a semi-silvered mirror placed in front of the eyes. A lends fixed above the half-mirror reflecs the beam of the light and transmits it to an optical system. This image, which follows eye movements, is called an "eye mark". The eye mark is superimposed on the image of the visual scene shown by a second lens placed between the eyes. The position of the eye mark is adjusted so that it appears in the visual scene on the exact spot at which the subject is gazing. Both the superimposed eye mark and image of the visual scene are recorded through an optical fiber by a $16 \mathrm{~mm}$. movie camera. The limits of the visual field recorded by this equipment are $22.7^{\circ}$ vertically and $31.4^{\circ}$ horizontally.

Improvements in this Eye Mark Recorder over previous models (Mackworth and Mackworth ${ }^{12}$ 1958, Shackel ${ }^{16}$ 1960, Mackworth, and Thomas ${ }^{13}$ 1962) are: 1) The half-mirror From the Department of Otolaryngology School of Medicine, Kyoto University, Kyoto 


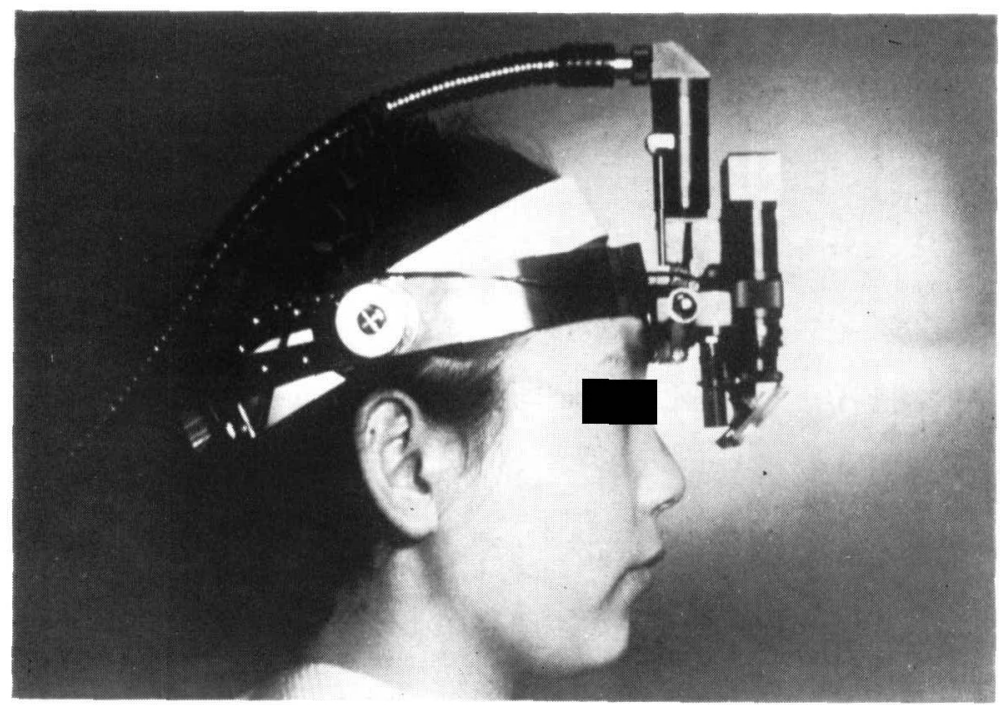

Figure 1. The Eye Mark Recorder.

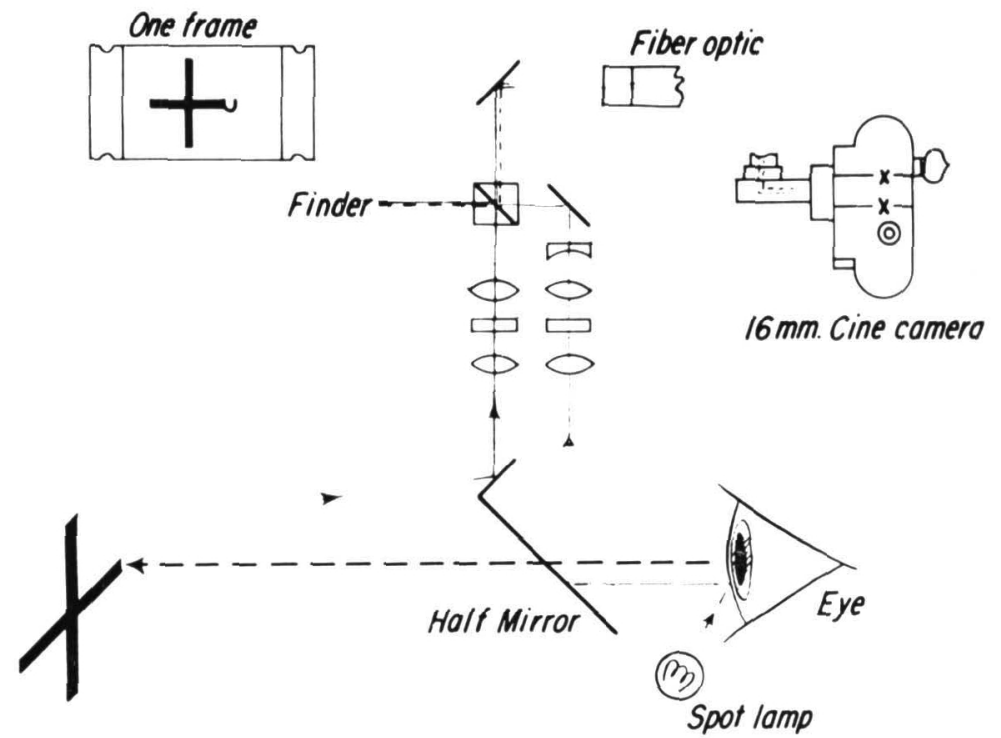

Figure 2. A schematic diagram of the Eye Mark Recorder.

is placed in front of the eyes so that natural binocular vision is possible. 2) The weight of the equipment (1.5 Lb.) is relatively light. 3) The scene lens is placed between the eyes, reducing parallax between the scene shown by the lens and that viewed by the eyes.

Eye movements with respect to the orbit were determined by measuring the movements of the eye mark relative to the frame of the film. Eye movements in relation to the object in the visual field were determined directly by measuring the movements of the eye mark with respect to the object on the film. Calibration of the position of the eye mark was carried out before every experiment.

2. Optical cylinder:

Equilibrium Res Suppl. 2 
In order to elicit optokinetic nystagmus, an electrically driven optical cylinder was used in this study. The cylinder was 36 inches in diameter and 41 inches in height. On its black surface, any of the following numbers of $3 / 4$ " wide white stripes could be attached at equal vertical intervals, to give various frequencies of stimuli at any given speed: $3,4,6,8,9,12,16,24$ and 42 . The angular speed of the cylinder could be adjusted to any value from $0^{\circ}$ to $200^{\circ} / \mathrm{sec}$. Those used in this study were $15^{\circ} / \mathrm{sec}$., $30^{\circ} / \mathrm{sec}$., $601 / \mathrm{sec}$., $90^{\circ} /$ sec., and $120^{\circ} / \mathrm{sec}$. Thus, for instance, when the optical cylinder was rotated with 12 lines at the rate of $60^{\circ} / \mathrm{sec}$, two lines passed in front of the subject each second.

3. Materials and procedure:

Ten normal healthy adults were used in this study. Each subject was placed at a distance of 18 inches from the surface of the optical cylinder, and was asked to follow each line without skipping (as best he could) as it passed in front of him. The cylinder was rotated clockwise and then counterclockwise. Eye movements for each combination of the various velocities and frequencies of lines (see the results) were recorded for periods of 20 seconds by using the Eye Mark Recorder ( 24 frames/sec.). Then, the records were analyzed.

\section{RESULTS}

1. Visual fixation patterns characteristic of optokinetic nystagmus:

Several different patterns of optokinetic nystagmus were classified according to the correlation between the gaze point and moving lines. Representative instances of these patterns are shown schematically in Figure 3.

a) With respect to the slow phase, nystagmus was differentiated into two patterns. In frames $2-30-1$ to $2-30-4$, the eye mark was fixed on the line as it moved to the left. In frame $2-30-5$, the eye mark slid a little from the line. The next frame, 2-30-6, indicates the rapid phase of nystagmus. In frame 2-30-7, the eye mark coincided perfectly with the next consecutive line. Then it moved to the left again fixed on the line. Since the line of sight was fixed on the object during the slow phase of nystagmus, this type of nystagmus was referred to as the foveal pattern. On the other hand, in frames $1-30-5$ to 1 $30-7$, and $4-120-6$ to $4-120-9$, the eye mark was completely off the line. In the series beginning 4-90-1, the eye mark failed to keep up with the line after having caught it at the beginning of the slow phase. These types of nystagmus were referred to as the retinal pattern, as foveal fixation did not occur. The amplitude of the retinal pattern was smaller than that of the foveal pattern when skipping (described later) did not occur.

b) Four types of movement of the eyes returning from one line to the next were observed, in addition to the hit form 2-30-7, in which the eye mark fixed on the next line perfectly at the end of the rapid phase. These four are referred to here as the waiting, overshooting, auxilliary, and skipping forms. In the overshooting form, the eye mark overshot the line $1-30-45$, then merged with it. In the waiting form, the eye mark stopped before reaching the next line 1-30-25, and then merged with it. In the series beginning with 1-30-1, the eye mark stopped before reaching the next line, and moved to the left at 

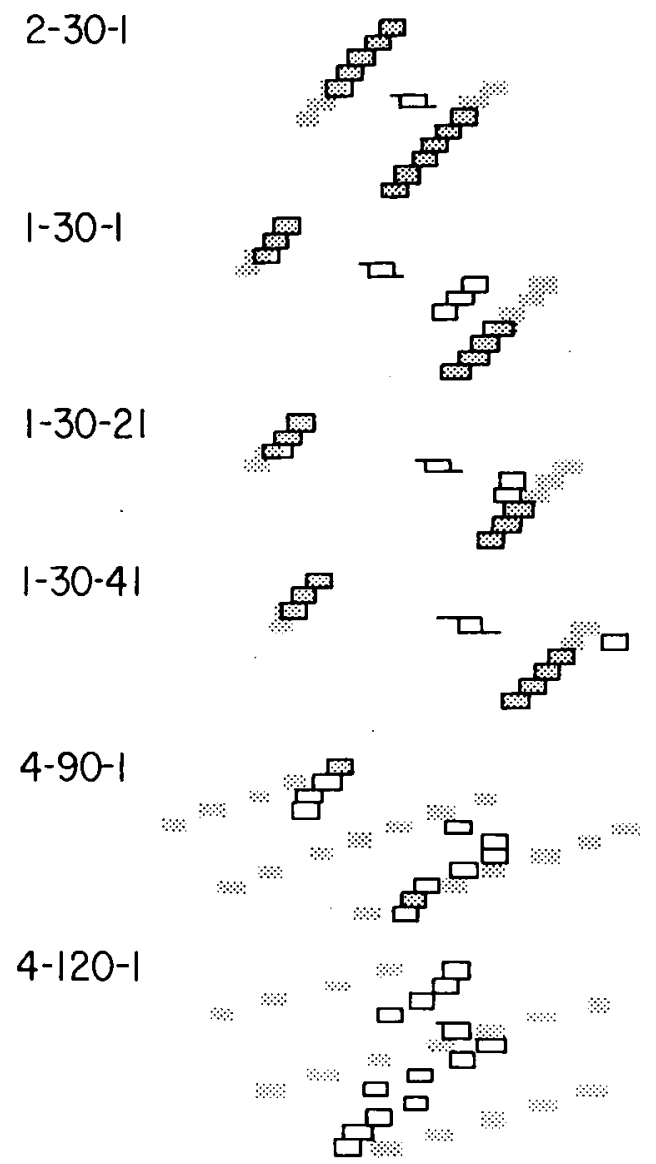

Figure 3. Various visual fixation patterns of optokinetic nystagmus: the simplified frames which just indicate positions of the gaze points (white squares) and lines (shaded squares), are arranged vertically in the order of their occurrance. The number which appears to the left of each series designates the frequency of lines (left), the velocity ot the cylinder (middle), and the proper number of the first frame. For example, number 2-30-1 indicates that nystagmus shown in this series was elicited at the stimulus frequency of $2 / \mathrm{sec}$. and the velocity of $30 \% \mathrm{sec}$. The -1 indicates the first frame.

the same rate as the line. Then it moved to the right again until it caught the line causing another beat of nystagmus. The amplitude of this form of nystagmus was obviously smaller than that of the foveal pattern when the two were seen together. When the eye mark bypassed one or more lines during the rapid phase of nystagmus, 4-90-4 to 4-90-6, it was called the skipping form. In skipping, the eyes usually made one smooth saccadic movement. Occasionally, however, this movement was interrupted by a momentary stop $(0.1$ sec.).

The auxilliary form of nystagmus was considered to be the retinal pattern because the eye mark was completely off the line during the slow phase. In the case of the waiting or overshooting forms, if the degree of waiting or overshooting was small and moment-

Equilibrium Res Suppl. 2 


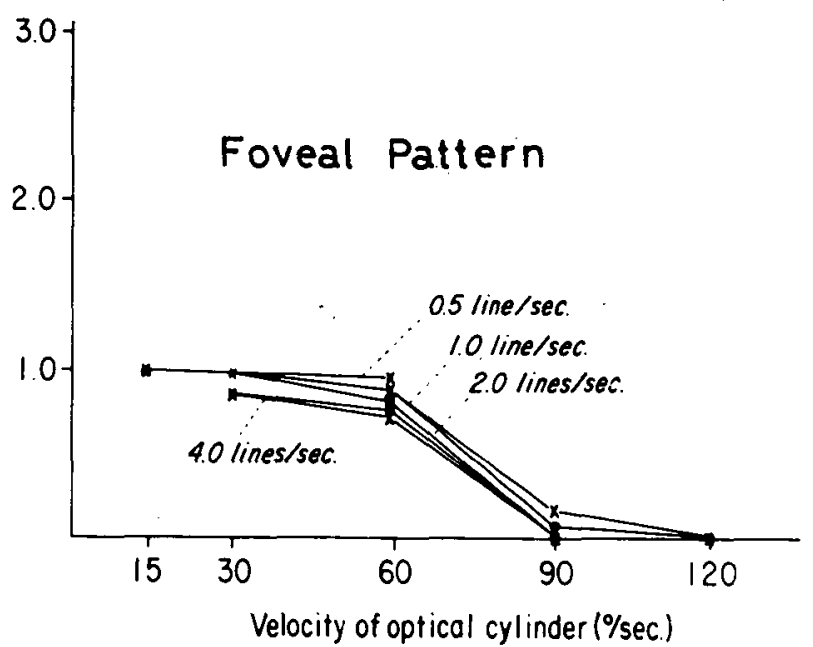

Figure 4. The incidence of the foveal pattern of nystagmus: The average numbers of foveal nystagmus beats per one line traversing in front of the subject are shown at various stimulus frequencies and velocities. Cross mark: Clockwise rotration of the optical cylinder. Dot mark: Counteclockwise rotation of the optical cylinder.

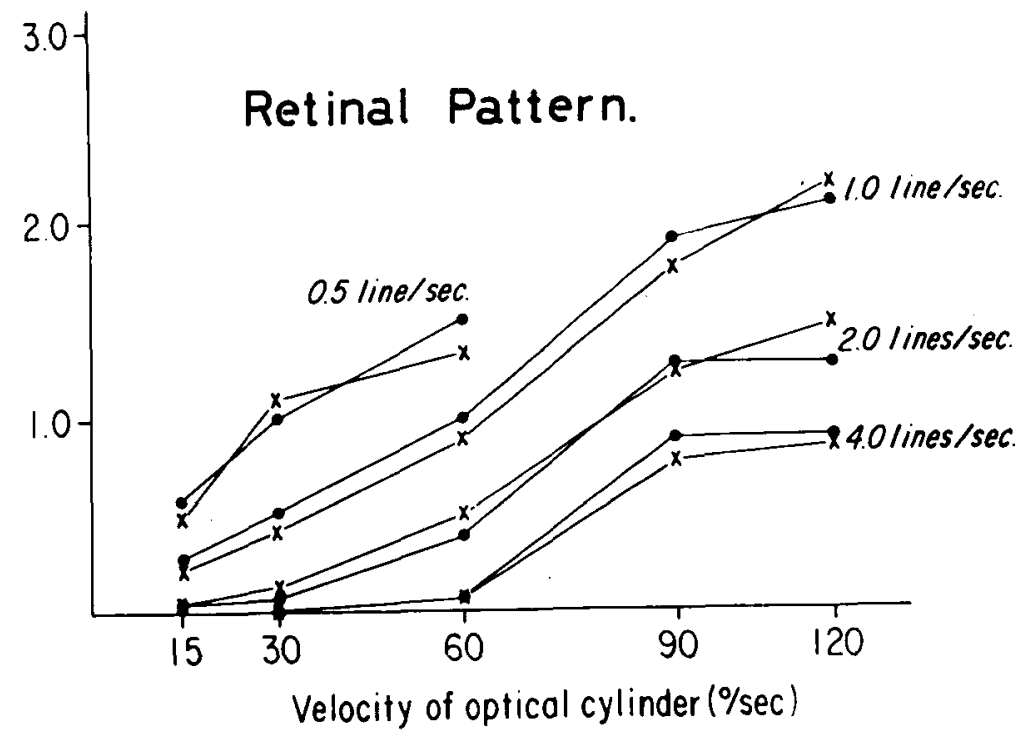

Figure 5. The incidence of the retinal pattern of nystagmus.

ary as shown in the series starting from $1-30-21$ or $1-30-41$, the subsequent slow phases were regarded as forming the foveal pattern. On the other hand, if the degree was neither small nor momentary, the subsequent slow phases was regarded as forming the retinal pattern (frames 4-90-6 to 4-90-11). The overshooting form in which the subsequent slow phase could be regarded as the retinal pattern was not actually observed.

2. Incidence of the patterns at various velocities and frequencies of optic stimuli. The incidence of foveal and retinal patterns is shown in Figures 4 and 5 respectively. 
The foveal pattern of nystagmus occurred when the cylinder was rotated at angular speeds below approximately $60 \%$ sec. The incidence of the foveal pattern was one per line at line frequencies below $2 \% \mathrm{sec}$. The incidence was decreased however at the relatively high velocity of $60^{\circ} / \mathrm{sec}$. This was due to the fact that the foveal pattern changed to the retinal pattern with the slowing of pursuit during the slow phase of nystagmus. The decrease of the incidence was greater at higher line frequencies than at lower ones. The incidence also decreased at a line frequency of $4 / \mathrm{sec}$. with the appearance of the skipping form.

The retinal pattern of nystagmus was observed at all angular speeds of the optical cylinder. The incidence of the retinal pattern per line increased with an increase in cylinder velocity as long as the line frequency was kept constant. When the velocity of the cylinder was kept constant, the incidence increased as the line frequency decreased. At velocities of the cylinder below $60^{\circ} / \mathrm{sec}$, the auxilliary form of nystagmus was seen except for that which had been changed from the foveal to retinal pattern by the slowing of pursuit, as previously mentioned.

When the angular speed of the cylinder was below $60^{\circ} / \mathrm{sec}$, the waiting and overshooting forms accounted for $5 \%$ or less of all nystagmus of the feveal pattern and the skipping form was negligible (less than $0.5 \%$ ) at line frequencies less than $2 / \mathrm{sec}$. At a line frequency of $4 / \mathrm{sec}$., the waiting and skipping forms increased to $10-21 \%$ and $11-25 \%$ respectively, while the overshooting form remained at or below $5 \%$. The degree of waiting was small and momentary.

When the angular speed of the cylinder increased beyond $60^{\circ} / \mathrm{sec}$. nystagmus patterns become disordered. The retinal pattern of nystagmus seemes to consist of the auxilliary form, and seems to be due either to the slowing of pursuit or to an increase in the degree of waiting. However, it was often difficult to distinguish between the two (4-120).

No significant difference in response was observed between clockwise and counter clockwise rotation of the cylinder.

In this experiment, the lines attached to the surface of the optical cylinder were actually stripes $3 / 4$ inches wide. It was possible therefore, for a single stripe to act as two. lines in that the subject could look from one edge of the stripe to the other; however, incidence of this was so rare as to be negligible.

\section{COMMENTS}

In 1936 ter Braak classified optok]netic nystagmus into two types, i.e., subcortical and cortical nystagmus. He thought that by the fixation movements of subcortical optic nystagmus all the images in the moving visual environment were maintained on the same spots of the retina, whereas by the fixation movements of cortical optic nystagmus, as was the case in this experiment, the image of one moving object was brought to and retained on the fovea centralis. It was difficult, however, to determine in each induced nystagmus whether or not the image of the moving object was retained on the fovea.

By superimposing the gaze point indicator on the image of the moving objects, in 


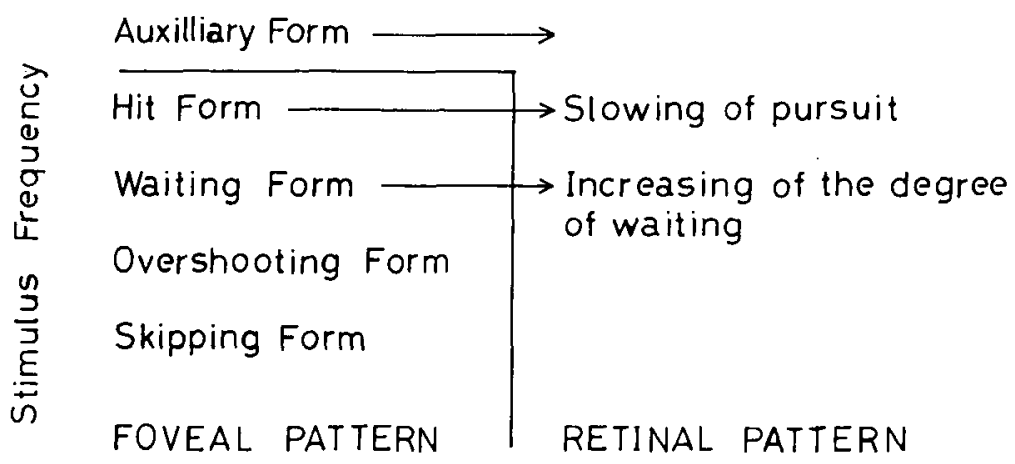

Figure 6 . The relationship between various types of nystagmus and optical stimuli.

this study, several types of nystagmus involving both foveal and retinal patterns actually were distinguished. Among them the foveal pattern of the hit form is considered to be the most adequate for visual adjustment. This pattern is commonly elicited at a stimulus frequency of $2 / \mathrm{sec}$. and at velocities below approximately $60^{\circ} / \mathrm{sec}$. Waiting and overshooting forms can be regarded as rudiments of transmutation to inadequate forms of nystagmus. That is to say, at low speeds of optical stimuli, rudimentary forms of inadequate adjustment patterns first appear at the end of the rapid phase of nystagmus. Actually, with an increase in the stimulus frequency the skipping form of nystagmus appears, whereas with a decrease in the frequency, the auxilliary form of nystagmus is prevalent, showing the inadequacy of the response. Although auxilliary nystagmus accompanied by the foveal pattern of nystagmus might not disrupt clear vision very much, since images of the objects are stabilized on the retina, it is noticed that the foveal pattern of nystagmus coexists with heterogeneous auxilliary nystagmus at low speeds and frequencies of stimuli. At velocities above approximately $60^{\circ} / \mathrm{sec}$. the foveal pattern of nystagmus changes to the retinal pattern either by slowing the pursuit or increasing the degree of waiting. This tends to make the visual images of the objects blurry throughout their course in the field of vision (Fig. 6.)

Thus, by taking the above described characteristics into consideration, not only could the adaptability of optokinetic nystagmus be evaluated properly, but also it's complexities could easily be understood. Regarding nystagmus rhythm, for example, Demetriades believed that it depended both on the distance between the stripes and the speed of the cylinder (1921). On the other hand, Ehler ${ }^{3}$ demonstrated that even if the number of stripes on the optical cylinder decreased, the frequency of optokinetic nystagmus did not decrease proportionately. He concluded that the nystagmus rhythm depended entirely upon the speed of the optical cylinder (1926). According to the present experiment, at higher stimulus frequencies, the skipping form began to appear, so that the increase in nystagmus frequency was not proportional to that of the stimulus frequency. Whereas, at lower stimulus frequencies, the auxilliary form of nystagmus appeared; the decrease of nystagmus fre- 
quency was then not proportional to that of the stimulus frequency. Therefore, the rhythmic discrepancy between stimulus and response can be attributed to the incidence of auxilliary and skipping forms of nystagmus. Since the auxilliary form of nystagmus is considered to be a refixation movement of the eyes as described by Dodge (1931), high frequency nystagmus due to frequent occurrence of the auxilliary form can not be regarded as an indication of adequacy, although it is generally so regarded. On the other hand, high frequency nystagmus due to the less frequent occurrence of the skipping form can be adequate. The amplitude of the foveal pattern of nystagmus is larger than that of the retinal pattern. It may be said that nystagmus with large amplitudes is more adequate than that with small ones if the skipping form does not occur. When the foveal pattern of nystagmus coexists with the auxilliary, they are usually easy to identify according to their amplitudes. But, when the retinal pattern takes the place of the foveal pattern of nystagmus either by slowing the pursuit or by increasing the degree of waiting, differences between beats of nystagmus become smaller in every respect, and the nystagmus looks homogeneous. Homogeneity of nystagmus may therefore not be a definitive sign of adequacy.

In conclusion, the adaptability of optokinetic nystagmus may be evaluated according to various parameters such as frequency, amplitude, and the like. However, it should be noted that misinterpretation is possible if the eye movement is considered apart from its relationship with visual objects.

\section{SUMMARY}

Optokinetic nystagmus was analyzed in ten normal subjects by superimposing a gaze point indicator on the image of moving objects. The foveal pattern of nystagmus was commonly elicited at stimulus velocities below approximately $60^{\circ} / \mathrm{sec}$. The retinal pattern was observed at all rates. At low stimulus speeds, rudimentary forms of inadequate adjustment such as the waiting or overshooting forms appeared. These changed to the auxilliary or skipping forms of nystagmus, indicating direct influence of stimulus frequency on inadequate response. At high stimulus speeds, the foveal pattern of nystagmus was changed to an inadequate retinal pattern by the slowing of pursuit or the increasing of the degree of waiting.

\section{REFERENCES}

1) BraAK, W.G. TER: Untersuchung uber optokinetischen Nystagmus. Arch. Neerl. Physiol. 21 : 309-376, 1936.

2) Dodge, R., Travis, R.C., and Fox, J.C.: Optokinetic nystagmus. 1II. Characteristics of the slow phase. Arch. Neurol. and Psychiat. 24: 21-34, 1930.

3) Ehiters, H.: On optically elicited nystagmus. Acta Ophthal. 3: 254-271, 1926.

4) Enocksson, P.: Optokinetic nystagmus in brain lesions. Acta Ophthal. 34: 163-184, 1956.

5) Fox, J.C., and Holmes, G.: Optokinetic nystagmus and its value in the location. Brain 49: $333-371,1926$

Equilibrium Res Suppl. 2 
6) Fox, J.C., Couch, F.H., ANd Dodge, R.: Optokinetic nystagmus, IV, Psychologic conditions. Arch. Neurol. and Psychiat. 26: 23-35, 1936.

7) CrutTner, R.: Experimentelle Untersuchung uber den optokinetischen Nystagmus. z.f. Sinnesphysiol. 68: 1-48, 1939.

8) Kitahara, M.: Eye mark recording as a vestibular test Related oculomotor reflex. Third symposium on the role of the vestibulra organs in space exploration. NASA $S p-152,403-$ $408,1967$.

9) Mackensen, C.: Untersuchungen zur Physiologie des optokinetischen Nystagmus. Albrecht V. Graefes, Arch. Ophthal. 155: 284-313, 1954.

10) Mackensen, G.: Untersuchungen zur Physiologie des optokinetischen Nystagmus. Albrecht v. Graefes, Arch. Ophthal. 156: 535-546, 1955.

11) Mackensen, G., And Uber, J.: Untersuchungen zur Physiologie des optokinetische Nystagmus, Albrecht v. Graefes, Arch. Ophthal. 161 : 599-604, 1960.

12) MAckworth, J.F., AND MACKWORTH, N.H.: Eye fixations recorded on changing visual senses by the television eye-marker. J. Opt. Soc. Am. 48:439-443, 1958.

13) Mackworth, N.H., and Thomas, E.L.: Head mounted eye-marker camera. J. Opt. Soc. Am. 52: 713-717, 1962.

14) Онм, J.: Die elementaren Grundlagen des optokinetischen Nystagmus, Albrecht v. Graefes Arch., Ophthal. 158: 485-494, 1957.

15) Rademaker, G.G.J., AND BraAk, W.G. Ter: On the central mechanism of some optic reactions, Brain. 71: 48-76, 1948.

16) Shackel, E.: Note on mobil eye view point recording. J. Opt. Soc. Am. 50: 763-769, 1960

(Accepted March 1, 1971) 